\title{
La interactividad de los dispositivos móviles geolocalizados, una nueva relación entre personas $\mathrm{y}$ cosas
}

\author{
Javier Fombona CADAVIECO \\ Universidad de Oviedo \\ fombona@uniovi.es
}

\begin{abstract}
Resumen
Este trabajo analiza las TICs móviles que utilizan los jóvenes, la tipología y características de estos equipos, según una muestra cercana a los 2000 alumnos de enseñanzas medias. Uno de los avances más interesantes es el posicionamiento por GPS o por el identificativo IP a través de la propia conexión Internet. Esta opción tecnológica nos permite hacer una propuesta para la interacción entre el usuario y su posición geográfica, también posibilita la descarga automática de información u otra actividad asociada a un lugar específico.
\end{abstract}

Palabras clave: Comunicación ubicua, Dispositivo móvil, Geolocalización, Realidad Aumentada, Smartphone, TIC.

\section{Geolocation for interactive mobile devices, a new relationship between people and things.}

\begin{abstract}
This paper analyzes mobile ICTs used by young people and the main characteristics of these teams, from a sample of 2000 students of intermediate education. One of the most interesting issue about this technology is the GPS positioning, which is independent of Internet or the IP identification. These technological possibilities has allowed us to make a software design for the interaction between the user and his geographical position, and it also enables automatic information downloading about the place, or other activity associated with a specific position.
\end{abstract}

KeyWords: Augmented Reality, Geolocation, ICT, Mobile Device, Smartphone, Ubiquitous Communication.

Referencia normalizada:

Fombona Cadavieco, J. (2013) La interactividad de los dispositivos móviles geolocalizados, una nueva relación entre personas y cosas. Historia y Comunicación Social. Vol. 18 Nºspecial Octubre. Págs. 777-788.

Sumario: 1. Introducción; 2. Metodología; 2.1. Descripción de los equipos móviles del alumnado; 2.2. Software de posicionamiento para equipos móviles; 2.3. La tecnología de Realidad Aumentada; 3. Resultados; 3.1. Datos de los dispositivos móviles del alumnado; 3.2. Opciones del software de posicionamiento para el docente; 4. Propuestas de actuación con dispositivos móviles; 5 . Conclusiones; 6. Referencias bibliográficas. 


\section{Introducción}

Los fenómenos relacionados con las Tecnologías de la Información y Comunicación (TIC) y las redes informáticas son controlados por grandes empresas de comunicación y desde al ámbito académico sólo podemos realizar intervenciones limitadas sobre sus consecuencias en las personas, no siempre positivas (Caldevilla, 2010) y dentro de un complejo mundo virtual que genera nuevos escenarios de relación que Mut Camacho (2012) denomina "reputación virtual".

En los últimos años los dispositivos móviles se han convertido en un elemento tecnológico de alto impacto en todas las sociedades y no sólo por su poder comunicativo. Los ciudadanos utilizan teléfonos portátiles en cualquier momento y lugar, pero su versión avanzada basada en los teléfonos inteligentes, o Smartphones, los ha convertido en una computadora de bolsillo que permite hacer muchas funciones. Estas opciones, junto a su penetración universal, hacen necesario que busquemos beneficios sociales, educativos y culturales. Especialmente en los países desarrollados estos recursos, además de ser un sistema de comunicación, se están incorporando al contexto académico en actividades para el desarrollo de determinadas competencias (Vázquez, Sevillano y Méndez, 2011). Recientes investigaciones han explorado su potencial pedagógico y diferencian los usos posibles y deseables (Nussbaum, 2007; Gil, Andersson, y Milrad, 2010; Xiaoyan, Ruimin, y Minjuan, 2007; Ramírez, Muñoz, y Delgado, 2008).

Pero continúa siendo preciso analizar el impacto que están teniendo esta versión portátil de las TIC. El trabajo aquí aborda esta temática desde una perspectiva y diseño interinstitucional, y recoge la experiencia de casi dos mil jóvenes participantes con el objetivo de aportar a la sociedad elementos de progreso cultural como contrapunto a los meros intereses económicos o a las estrategias de marketing que puedan rodear a estas tecnologías.

El hecho de la inserción de la telefonía móvil en nuestra sociedad la convierte en el soporte para el acceso de tecnologías como la Geolocalización, y su potencial integración con los desarrollos de Realidad Aumentada. Estas aplicaciones asocian información a un lugar dado o a un objeto específico, posteriormente el usuario del dispositivo móvil recibe esos datos cuando se sitúa dentro de un radio de determinada proximidad al lugar fijado. Las posibilidades de interacción son múltiples y muy potentes, hasta el punto de poder tener controlada la posición y movimientos del usuario. La persona que crea estas actividades puede ser variada, un docente, un guía turístico o simplemente alguien que guía las actividades de campo de otras personas (Perales y Adem, 2013). Para ello se pueden considerar diversos desarrollos existentes u otros nuevos, y organizar sus estrategias teniendo en cuenta las habilidades asociadas a estos equipos.

Las técnicas de geo-posicionamiento son utilizadas comúnmente en dispositivos de navegación y orientación geográfica de los conductores de vehículos, en equipos de orientación en senderismo y escalada, o en los sistemas de brújula y posición 
geográfica incorporados a muchos los teléfonos celulares avanzados. Lo interesante de esta técnica es su conexión con un entorno concreto o con objeto reconocible y capaz de disparar una información almacenada digitalmente, e incorporar datos a la realidad, la Realidad Aumentada. La característica diferencial es la descentralización espacio/temporal, la ubicuidad en los lugares y momentos de gestión de la información. Esta circunstancia junto a la universalización de su uso, hace que los dispositivos móviles sean el elemento tecnológico que mayor impacto tiene en todas las sociedades por su proyección futura.

Por otro lado, y junto a los diversos elementos que conlleva el uso de estas tecnologías, destaca la trascendencia que los dispositivos móviles tienen por su elevado potencial motivador en los jóvenes, y aquí está una de las claves en la estrategia de penetración de los medios, de las tecnologías y de las TIC en sus usuarios. El hecho de utilizar un dispositivo móvil para transmitir un texto, o un mensaje tradicional es una circunstancia motivadora, y podemos acercarnos a una nueva metodología educativa si aplicamos los últimos desarrollos en software para equipos. En este sentido, dos de estas aplicaciones un tanto espectaculares se relacionan con estas posibilidades de Geolocalización y Realidad Aumentada (Fombona, Pascual y Amador, 2012).

\section{Metodología}

Esta investigación surge desde un diseño que contó con la participación de investigadores de varias universidades. Los datos aquí reflejados son parte del proyecto PPI 2012 de la Universidad de Oviedo "Desarrollo de la Realidad Aumentada sobre dispositivos móviles como apoyo a la acción educativa". El objetivo fundamental es aportar a la sociedad elementos de progreso cultural a partir de la utilización de las TIC en su versión dispositivo móvil, esto es, encontrar utilidades socio educativas a los teléfonos inteligentes o Smartphones que tiene el alumnado. Subrayamos que los centros educativos suelen prohibir o limitar la utilización de estos equipos ya que supone una desviación de la normalidad dentro del aula, y por tanto es necesario aportar soluciones que sean aceptadas por la comunidad académica (Fombona, Goulao y Fernández, 2012). Esta es una propuesta también contrasta con los simples intereses económicos o a las estrategias de marketing que normalmente rodean a estas TIC.

En el trabajo se plantearon dos fases una inicial en la que se explora y describe la tipología de TICs Dispositivos Móviles que los jóvenes tienen y utilizan de forma habitual, y una segunda fase en la que se hace una propuesta de utilización del software de Geolocalización.

\subsection{Descripción de los equipos móviles del alumnado}

En la primera fase se realizó en estudio descriptivo sobre los equipos que tiene el alumnado, y el objetivo específico consistía en descubrir la tipología y características de estos dispositivos. La muestra analizada consistió en 1868 alumnos/as 
de enseñanzas medias, con edades entre los 12 y 18 años, y que se encontraban en centros de Enseñanza Secundaria Obligatoria, Bachiller y Formación Profesional en la región española de Asturias. Como instrumento se construyó un cuestionario de respuesta anónima, cuya fiabilidad se obtuvo a partir de una validación inicial realizada por expertos que dimensionaron los contenidos, su precisión y complementariedad, también se realizó un ensayo previo para verificar la ausencia de errores. La validez y la generalización externa se apoyó en la selección aleatoria de la muestra, y también se hizo una elección de ítems sencillos para el test tal que no ofreciera mayores dificultades a los encuestados. Es importante destacar que el profesor/a aplicó el cuestionario dividiendo la muestra en los grupos naturales donde impartía docencia este control del docente en cada aplicación confieren validez al constructo. El cuestionario presenta el contenido que se describe a continuación:

- Ítem 1: Datos de Identificación del Centro, Ciudad, Titularidad.

- Ítem 2: Datos del grupo y nivel educativo encuestado (ESO, FP, Bachiller).

- Ítem 3: Número de alumnos analizados.

- Ítem 4: Número de alumnos con teléfono móvil/celular.

- Ítem 5: Número de alumnos del tipo BlackBerry.

- Ítem 6: Número de alumnos del tipo BlackBerry con GPS integrado.

- Ítem 7: Número de alumnos del tipo IPhone.

- Ítem 8: Número de alumnos del tipo IPhone con GPS integrado.

- Ítem 9: Número de alumnos con otro teléfono con sistema operativo Android.

- Ítem 10: Número de alumnos con otro teléfono con sistema operativo Android y con GPS .

- İtem 11: Número de alumnos con otro teléfono móvil sin sistema operativo.

- Ítem 12: Número de alumnos planean tener un teléfono móvil con GPS en los próximos meses.

- Ítem 13: Número de alumnos tienen una Tablet o Ipad con GPS.

\subsection{Software de posicionamiento para equipos móviles}

Las aplicaciones directas de la Geolocalización consisten en detectar la posición del equipo, situarlo en un mapa o en una vista desde satélite y relacionarlo con lugares próximos. Los aparatos con GPS (Global Positioning System) realizan esta función, que en ocasiones precisa del acceso o contratación de los servicios de un servidor de Internet para ofrecer en tiempo real los datos de la Longitud y Latitud en donde se encuentra el usuario, es decir, el aparato está sujeto a los servicios de una compañía de telefonía. En otras ocasiones el dispositivos gestiona estas coordenadas y ordenadas con una interacción independiente, tal y como lo hacen los dispositivos GPS de los vehículos. A partir de estos datos el equipo y el software correspondiente sitúan al usuario en un mapa previamente almacenado, que así mismo puede tener cargadas otras referencias, por ejemplo hoteles, museos cercanos u otros lugares (Sharples, 2007), como sucede con la aplicación Geo Layers. La persona que maneja estos equipos puede observar en la pantalla del dispositivo móvil una visión de su posición 
desde lo alto, desde un satélite, integrándola en las vistas que suministra un software como el de Google Maps.

También existe la posibilidad de integrar nuestra situación con lo que esté captando la cámara (Imagen 1), además de combinar la posición geográfica con un mapa o una visión satelital. Estas funciones están presentes en múltiples aplicaciones, tales como Layar, Juanio, Panoramio, Vision, etc., e incorporan informaciones diversas aunque predominan las de tipo comercial. Todas tienen interfaces similares con pautas de interacción con el usuario para localizar y guiar hasta un destino dado ofreciendo la distancia relativa y la posición a modo de brújula.

Imagen 1. Foto posicionando las coordenadas del lugar e insertando de datos relacionados

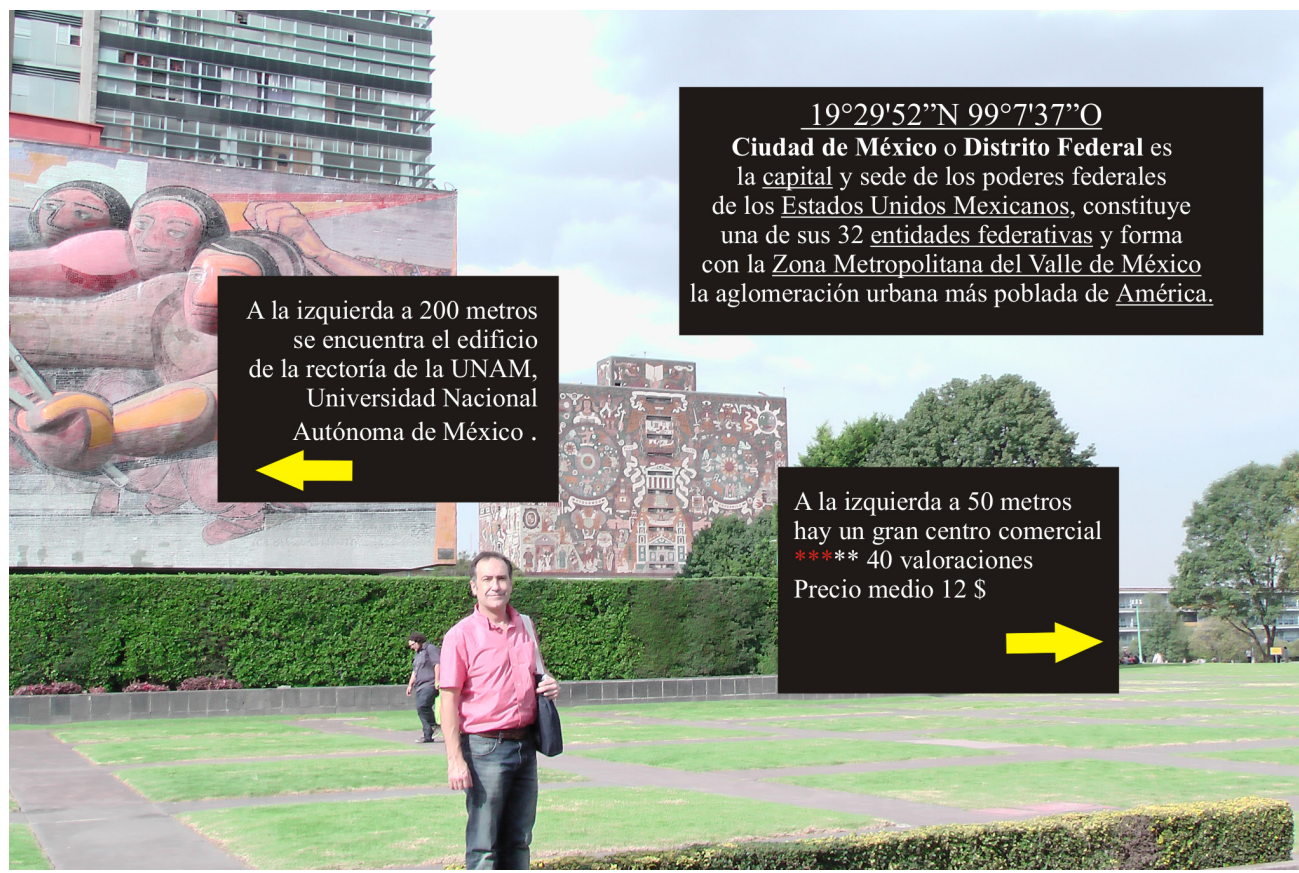

Fuente: Elaboración propia.

Aunque el posicionamiento por GPS puede ser independiente de la conexión a Internet, existen lugares en los que no hay acceso a los satélites, tales como las zonas cubiertas o el interior de los edificios, por lo que actualmente también existe una tecnología no tan precisa, que permite el posicionamiento por la dirección del Protocolo de Internet (IP) a través de una conexión Internet, es decir, a través de un programa que analiza los datos de IP y del propio proveedor de Internet. La IP es un número único asignado por los proveedores de servicios de Internet a cada ordenador conectado a Internet. En este sentido hay aplicaciones como AdWords que usan una 
serie de factores para determinar la ubicación física del ordenador o del dispositivo móvil de un usuario.

Por otro lado, si un dispositivo móvil está conectado a una red Wi-Fi y a un servidor proxy, podemos detectar la dirección IP para determinar la ubicación física. Eso sucede cuando los usuarios deciden compartir la ubicación precisa del dispositivo móvil. Por último se debe describir el cálculo de la ubicación aproximada mediante el uso de información sobre la ubicación de las torres de telefonía a las que un dispositivo móvil está conectado a en cualquier momento.

Una opción que contemple varias de estas posibilidades permite una localización del usuario más perfecta y la interacción con su posición geográfica, así como la descarga automática de información u otra actividad asociada a un lugar específico. A estas situaciones se une la necesidad de satisfacer la múltiple variedad de equipos, es decir, además de los aparatos que poseen la tecnología para la gestión del posicionamiento, también se debe contemplar aquellos otros que cuentan o no con un sistema operativo, o alternativas para los que solo tienen conectividad básica del tipo conexión Wifi o intercambio de datos por el sistema Bluethooth.

\subsection{La tecnología de realidad aumentada}

En la técnica de Realidad Aumentada un software reconoce una imagen patrón o un código de reconocimiento $(\mathrm{QR})$ captado por la cámara fotográfica del dispositivo móvil, esa imagen sirve para disparar otra información almacenada y que se superpone al patrón icónico captado (Imagen 2).

La tecnología de Realidad Aumentada se puede gestionar utilizando con el computador de sobre mesa tradicional, los dispositivos portátiles y con equipos específicos para la técnica de Realidad Aumentada tales como unas gafas de visión específica. El caso más sencillo es la gestión de Realidad Aumentada en computador tradicional al que se conecta una cámara para digitalizar la imagen captada, a la que se le incorporan capas con otros datos, más imágenes fijas, imágenes en movimiento, textos y/o sonidos. Esta información se añade a partir de un software que selecciona documentos específicos de una base de datos propia o del acceso a los datos de la red Internet. Una pantalla presenta la síntesis coordinada entre realidad y virtualidad, y muestra de forma sincronizada tanto las imágenes reales captadas, junto al resto de datos superpuestos sincronizados en tamaño, posición y en tiempo real.

La posibilidad de gestión de Realidad Aumentada en dispositivos móviles avanzados, Smarphones, es un ajuste del fenómeno descrito a estas pequeñas mini computadoras que incorporan cámaras de captura de imágenes. Con ello se superpone en la pantalla del dispositivo el resultado de Realidad Aumentada sobre la imagen de un objeto dado. 
Imagen 2. Foto que al captar y reconocer determinado patrón provoca la visualización de una animación almacenada y superpuesta.

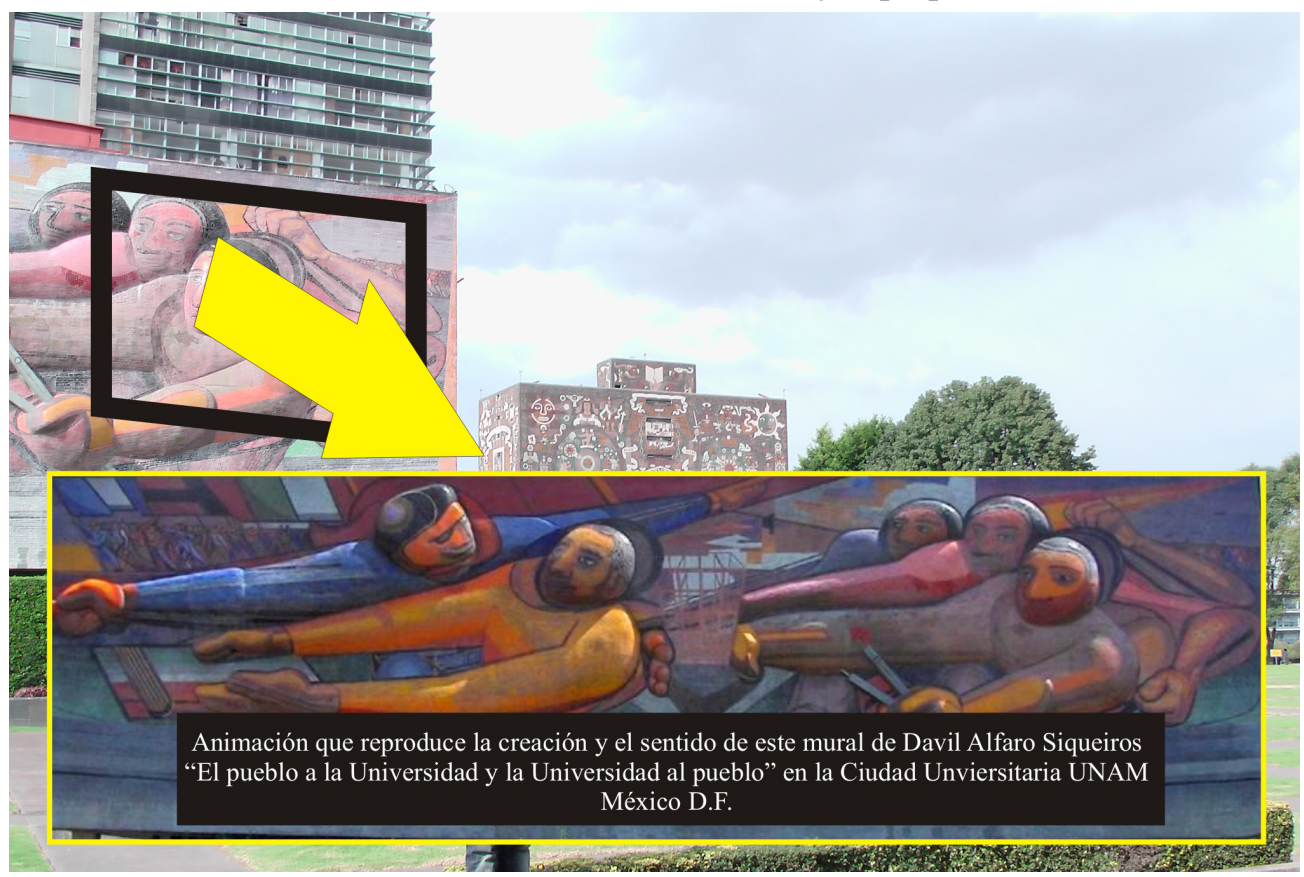

En ocasiones hay dispositivos específicos captura y visión que se integran en unas gafas especiales u otros dispositivos específicos y permiten al usuario ver la realidad a través de la lente y superponer y mostrar otra información gráfica. Esto suele utilizarse en empresas avanzadas de mantenimiento y reparación de maquinarias complejas que proporcionan estos aparatos especiales para combinar visión y las instrucciones correspondientes para que el operario realice la intervención con este apoyo. Estas aplicaciones pueden hacer que surja información de lugares específicos al ser captados con la cámara del dispositivo portátil del usuario, por ejemplo esta utilidad tiene actualmente desarrollos para museos o para textos ilustrados que son capaces de superponer otra información del tipo de texto, audio o de video (Perales y Adem, 2013).

\section{Resultados}

3.1. Datos de los dispositivos móviles del alumnado

Los resultados obtenidos sobre la muestra analizada que describe la tipología de los aparatos portátiles se muestran en el Cuadro 1. Cabe indicar que en el número 
total de alumnos encuestados, 1868, hay casos que poseen varios equipos portátiles por lo que los datos no son exactamente complementarios entre sí. Casi la totalidad de los alumnos, más del 95\%, manejan habitualmente un teléfono portátil, siendo en su mayor parte un equipo gestionado con el sistema operativo Android, un 40,5\%. Y un $68,2 \%$ del total de jóvenes tienen equipos con la tecnología GPS capaces de funcionar con aplicaciones de Geolocalización. A estos datos cabe añadir que 223 personas participantes en el estudio, un $11,9 \%$ se plantean adquirir un dispositivo móvil equipado con sistema de Geolocalización (ítem 12) por lo que la proporción de estos aparatos alcanzaría el $80 \%$.

Tabla 1: Nivel de implantación entre el alumnado de los distintos dispositivos móviles.

\begin{tabular}{|l|l|l|l|}
\hline Ítem & Dispositivo & $\mathbf{N}^{\mathbf{0}}$ & $\mathbf{\%}$ \\
\hline 4 & Cantidad de alumnos con dispositivo móvil. & 1780 & 95,3 \\
\hline 5 & Implantación de dispositivos Blackberry. & 480 & 25,7 \\
\hline 6 & Implantación de dispositivos Blackberry con GPS integrado. & 230 & 12,3 \\
\hline 7 & Implantación de dispositivos Iphone. & 118 & 6,3 \\
\hline 8 & Implantación de dispositivos Iphone con GPS integrado. & 116 & 6,2 \\
\hline 9 & $\begin{array}{l}\text { Implantación de dispositivos con sistema operativo (S.O.) } \\
\text { Android. }\end{array}$ & 756 & 40,5 \\
\hline 10 & Implantación de dispositivos con sistema S.O. y GPS integrado. & 595 & 31,8 \\
\hline 11 & Implantación de dispositivos móviles sin sistema operativo. & 354 & 18,9 \\
\hline 12 & Intención de tener un equipo con GPS & 223 & 11,9 \\
\hline 13 & $\begin{array}{l}\text { Implantación de dispositivos móviles tipo Tablet o Ipad con } \\
\text { GPS. }\end{array}$ & 335 & 17,9 \\
\hline
\end{tabular}

\subsection{Opciones del software de posicionamiento para el docente}

Los datos de los dispositivos móviles son una referencia sobre las posibilidades que desde el ámbito docente se deben explorar, sobre todo para conocer las opciones que ofrece la tecnología de Geolocalización en el contexto docente y poder sacar un rendimiento socio-educativo a esos equipos. Muchas de las aplicaciones específicas que trabajan con estas técnicas de posicionamiento son de distribución gratuita y se obtienen a través de los propios servicios de descarga de cada plataforma (Blackberry, Iphone o Android), en otros casos es necesario un reducido pago para obtenerlos. En todo caso, es preciso que algunas de estas dos circunstancias se den positivamente: existencia de una conexión a la red de datos Internet o estar en una zona de acceso WIFI con acceso a la señal de Internet, o estar en una zona con cobertura GPS.

En todo caso debemos ofrecer alternativas tanto para los usuarios con equipos geolocalizados como para quienes tienen dispositivos sin sistema operativo, el $18,9 \%$. Se debería dar una solución en el caso de que el equipo se encuentre o no 
tenga capacidad tecnológica de conexión a las redes de datos o al sistema GPS. En este sentido algunas aplicaciones, como el caso del Software Gardens, desarrollan las opciones tanto para utilizar el dispositivo móvil con las funciones de Geolocalización descritas en el mismo lugar donde van a ir ofreciendo las presentaciones de la información a partir de la técnica de Realidad Aumentada, y otra opción, para trabajar los mismos contenidos fuera de línea, en otro lugar, sin aprovechar la automatización en la presentación de los contenidos. Esto es, existe la posibilidad de explorar de la información off-line, fuera de la conexión directa con el lugar, en este caso aparecen los textos, audios o videos explicativos que se reproducen automáticamente en el modo on-line cuando el usuario está en ese lugar, y que se puede solicitar también off-line estando fuera de ese sitio.

\section{Propuestas de actuación con dispositivos moviles}

La persona que crea actividades para usuario de dispositivos móviles apoyado con la técnica de Geolocalización puede ser un docente o puede tener otro perfil muy diverso, por ejemplo puede ser un guía turístico, un asesor comercial o simplemente unos padres que quieren guiar las actividades de sus hijos. Se pueden diseñar tareas para usar estas tecnologías con el software existente de Google Maps, u otros específicos tanto para dispositivos móviles Iphone (Geolocation, Indicium-geolocation, MeetPoint, Directions, GeoPostr, etc.), como para equipos Android (Geolocation, AR Bearing,GeoPlum, Ubalda Geo, etc ), para BlackBerry (Geolocator), u OviMaps de Nokia.

Las actividades pueden ser todas aquellas que requieran una salida y exploración de campo, tales como reconocimiento de sitios, lugares, accidentes geográficos, etc. o sugerencias para seguir una ruta geoposicionada geográficamente al indicar los puntos por los que debe pasar el usuario, estas tareas pueden planificarse, por ejemplo, a través de Google Maps 3D o Street view que visualiza en el ordenador los datos geográficos y la imagen fotográfica del lugar. Quien diseña la actividad posiciona geográficamente sus informaciones, y por ejemplo, solicitar que identifiquen y describan el monumento que está en $42.599,-5.567$ para que el usuario sea llevado por el dispositivo móvil con GPS justo delante de la Catedral de León en España.

En el campo de la Realidad Aumentada puede utilizar referencias ya creadas con determinado software (AcrossAir, GoogleGoggles, Wikitude, Layar, Yel, Augment, Arsights, etc.). Algunas de estos ofrecen la posibilidad de que un usuario incorpore datos a determinadas imágenes captadas o patrones QR creados por el usuario, tal es el caso de Arsights que reproduce monumentos con movimientos en 3D sobre capturas realizadas por el usuario.

Pero también habría que ofrecer también alternativas para las personas sin dispositivo móvil avanzado. Y realizar una descarga previa de los datos en un modo de texto con gráficos y proceder a la respuesta por un método más tradicional derivado de una 
conexión a través de un cable de datos, envío por email, por acceso a una página Web o con un documento para su trabajo posteriormente en la computadora tradicional apoyados por la visualización de Google Maps. En todo caso no se puede confiar en que se vaya a disponer siempre de conexión a Internet o cobertura GPS, por lo que el software debería almacenar los documentos en un formato legible para su uso off-line. El modo on-line no debe ser un requisito obligatorio, pero sí resulta interesante su uso para obtener la respuesta y la realimentación inmediata entre alumno y docente. Por ejemplo, la comunicación de las respuestas por parte de los usuarios en el momento en que realizan las actividades ofrece al profesor una información directa de los resultados, permitiéndole actuar en consecuencia on-line: repetir la actividad si muchos alumnos han respondido erróneamente, proponer otro nivel de dificultad, etc. En el desarrollo que propone y realiza este grupo de investigación autor de este artículo, posibilita que un profesor introduzca una cuestiones para un destino a visitar en una actividad de campo por su alumnado y que los estudiantes vayan individualmente aproximándose al lugar y verificando la información que aparece una vez situados en determinadas posiciones concretos, y evitando la necesidad de guiar a cada persona específicamente hacia el objetivo de esa actividad.

Es importante resaltar el atractivo que tiene esta tecnología en su dimensión plena de posicionamiento y Realidad Aumentada, especialmente cuando las imágenes captadas por la cámara del dispositivo móvil provocan el disparo de la información almacenada previamente con una coherencia tridimensional tal que cualquier movimiento del aparato hace que tenga idéntica respuesta en el movimiento del la imagen superpuesta. El contexto educativo la Realidad Aumentada por si sola también puede enriquecer muchos de los contenidos de los libros incorporando documentos multimedia. También puede usarse para ver partes ocultas de un objeto de difícil acceso, o peligrosas para el aula, y que captamos con la cámara del dispositivo móvil y sobre la que se superpone la correspondiente información complementaria. Los efectos de esta realidad virtual superpuesta a la imagen real son tan sorprendentes que se están abriendo nuevas líneas de trabajo en la simulación e integración ambos componentes (Wu, 2013).

\section{Conclusiones}

En los niveles de enseñanzas medias, los aparatos de telefonía móvil se han convertido en los equipos que tienen más del $95 \%$ del alumnado. Los usos son comunicacionales y lúdicos, grabaciones de video y audio, conexiones a Internet y un largo abanico de aplicaciones. Siendo el uso específico docente muy reducido o casi nulo. Por ello, y apoyándonos en su potencial motivador, deberíamos de aprovechar sus opciones en determinados momentos educativos. Por ejemplo, el marco de las actividades de campo puede ser un escenario propicio para usar estos dispositivos como elemento gestor en las actividades formativas asociadas a la Geolocalización de las tareas. El profesor puede realizar previamente un diseño de la actividad con la 
visualización en Google Maps y planificación las tareas el lugar de la visita/excursión antes de que acudan los alumnos. Posteriormente los usuarios irán enfocando con el dispositivo móvil los lugares propuestos, y en ese momento les aparecerá la información geolocalizada en su teléfono móvil Smarphone. Estas técnicas que unen realidad y virtualidad, además de lograr la conexión del usuario con un lugar concreto (Geolocalización) y/o con objeto específico (Realidad Aumentada), pero son potentes ya que estos equipos mantienen un elevado nivel de atractivo para los jóvenes.

\section{Referencias bibliográficas}

CALDEVILLA, D. (2010). Internet como fuente de información para el alumnado universitario. Cuadernos de Documentación Multimedia, $\mathrm{n}^{\mathrm{o}}$ 21, Madrid: Universidad Complutense, pp.: 141-157.

FOMBONA, J.; PASCUAL, Á.; AMADOR, F. (2012). Realidad Aumentada, una evolución de las aplicaciones de los dispositivos móviles. En: PixelBit. Revista de Medios y Educación, $\mathrm{n}^{\circ}$ 4, Sevilla: Universidad de Sevilla, pp.: 207-220.

FOMBONA, J. GOULAO, F. y FERNÁNDEZ, A. (2012). Using Augmented Reality and m-Learning to Optimize Students Performance. En: Higher Education Procedia - Social and Behavioral Sciences, $\mathrm{n}^{\circ}$ 46, Barcelona, pp.: 2970-2977.

GIL, D.; ANDERSSON, J. y MILRAD, M. (2010). "Enhancing mobile learning activities by the use of mobile virtual devices - some design and implementation issues". En 2nd Int. Conference on Intelligent Networking and Collaborative Systems. Thessaloniki, Grecia, pp.: 137-144.

MUT, M. (2012). La desvirtualizacion del concepto reputación en el entorno virtual. En: adComunica. Revista Científica de Estrategias, Tendencias e Innovación en Comunicación, $\mathrm{n}^{\mathrm{0}} 3$, Castellon: Asociacion para el Desarrollo de la Comunicación adComunica, Universidad Complutense de Madrid y Universitat Jaume I, 227-229. DOI: http://dx.doi.org/10.6035/2174-0992.2012.3.18

NUSSBAUM, M. (2007). "Comparative study of peer learning mediated by interconnected PCs and PDAs", En 6th Annual International Conferences on Mobile Learning, Melbourne, pp.: 194-198.

PERALES, V. y ADAM, F. (2013) Integratiton of GIS (Geographic Information System) and locative tools in pedagogical and ludic practices for museums. En: Arte Individuo y Sociedad, $\mathrm{n}^{\mathrm{0}}$ 25, Murcia: Univ. Murcia, pp.: 121-133.

RAMÍREZ, G.; MUÑOZ, M.; DELGADO, C. (2008). "Exploring touching learning environments". En KENDALL, M. y B. SAMWAYS, B. (ed.) (2008). Learning to live in the knowledge society, International Federation for Information Processing, $\mathrm{n}^{\circ}$ 281, Boston: Springer, pp.: 93-96.

SHARPLES, M. (2007). "An evaluation of my artSpace: a mobile learning service for school museum trips". En NORMAN, A. y PEARCE, J. (ed.). 6th Annual Conference on Mobile Learning, mLearn. Melbourne: University of Melbourne, pp.: 238-244. 
XIAOYAN, P.; RUIMIN, S. y MINJUAN, W. (2007). "Building learning communities in blended classrooms through an innovative mLearning system". En Virtual Environments, Human-Computer Interfaces and Measurement Systems (2007), pp.: $139-143$.

SEVILLANO, M. y VÁZQUEZ, E. (2008). Potencialidad de las tecnologías de la información y comunicación en las labores de gestión educativa. En: Avances en Supervisión Educativa, $\mathrm{n}^{\circ}$ 8, Madrid: ADIDE.

VÁZQUEZ, E.; SEVILLANO, M.; MÉNDEZ, M. (Coords.) (2011). Programar en Primaria y Secundaria. Madrid: Pearson.

WU, H.; et al. (2013). Current status, opportunities and challenges of augmented reality in education. En: Computers \& Education, $\mathrm{N}^{\mathrm{o}}$ 62, Oxford: Pergamon-Elsevier Science, pp.: 41-49.

\section{El autor}

Javier Fombona. Además de su función como coordinador y dinamizador de redes y convenios internacionales, sus trabajos abordan principalmente la incorporación de las Nuevas Tecnologías Audiovisuales Aplicadas a la Educación. Ex-realizador de TV, profesor en el área de la Didáctica y la Comunicación Audiovisual desde 1986 en distintos niveles educativos; ha impartido múltiples comunicaciones, conferencias y publicaciones sobre estos temas, también es revisor de artículos para varias revistas científicas internacionales. Investiga la inserción de la narrativa audiovisual en los nuevos soportes tecnológicos. Y actualmente coordina varios proyectos de investigación sobre Dispositivos Móviles Geolocalización y Realidad Aumentada. 